\title{
EPIDEMIOLOGY AND AETIOLOGICAL DIAGNOSIS OF MICROBIAL KERATITIS: GUIDELINES FOR ITS TREATMENT
}

\section{Sridevi ${ }^{1}$}

${ }^{1}$ Associate Professor, Department of Ophthalmology, Rajah Muthiah Medical College \& Hospital, Annamalai Nagar, Chidambaram.

ABSTRACT
BACKGROUND
Globally, it is estimated that ocular trauma and corneal ulceration result in $1.5-2$ million cases of corneal blindness annually. $90 \%$
of such patients hail from developing countries, where the lesion has now been recognised as a silent epidemic. A National survey
by the Government of India (1991-2001) estimated that corneal lesions were responsible for $9 \%$ of blindness in the country. The
aim is to study various factors in epidemiology and aetiological diagnosis of suppurative keratitis in 50 patients attending the
Department of Ophthalmology, Rajah Muthiah Medical College and Hospital during April 2015 to October 2016.

\section{MATERIALS AND METHODS}

This study was undertaken during the period from April 2015 to October 2016. 50 patients of corneal ulcer attending Ophthalmic Outpatient Department of Rajah Muthiah Medical College and Hospital were the subjects of this study. Visual acuity was recorded using Snellen visual acuity chart. Syringing was done to determine the patency of the nasolacrimal duct. The IOP was estimated by using digital pressure above the tarsal plate. Epithelial defect was demonstrated by fluorescein staining the size of the corneal ulcer and the level of the hypopyon is measured with slit lamp.

Design- Prospective, observational study.

\section{RESULTS}

In this study of 50 cases of corneal ulcer, the following results were obtained. The percentage of fungal keratitis is $54 \%$ proved clinically and by culture and sensitivity while that of bacterial keratitis is $46 \%$. The majority of patients were found in the Age group of less than 50 years, male patients showed higher prevalence than female patients. Most of the patients were agriculturists (64\%). Right eye was affected more in patients (66\%) due to right handedness of the individual. $64 \%$ of the patients presented with history of injury with stick (24\%), paddy husk (12\%) and thorn (12\%). 24\% of patients used topical medications like antibiotics and steroids prior to reporting. $86 \%$ of patients attended our OPD within 5 days of injury. The rest reported after 10 days. The size of the ulcer measured up to $4 \mathrm{~mm}$ in $94 \%$ of patients. The nasolacrimal duct was patent in $90 \%$ of patients. The most common bacteria identified was Staphylococcus 16\% and Pneumococci 33\%. As far as other eye is consent 54\% were normal 20\% showed lens changed and $10 \%$ were pseudophakics. The fungi identified were Aspergillus $27 \%$ and Fusarium $73 \%$.

\section{CONCLUSION}

Treatment success is considered if there is complete healing of the ulcer within 2 weeks after starting therapy. Most of the patients came in advanced stages of the disease due to negligence, poor economic condition, ignorance, blind faith in native treatment.

\section{KEYWORDS}

Aetiological Diagnosis, Microbial Keratitis.

HOW TO CITE THIS ARTICLE: Sridevi V. Epidemiology \& aetiological diagnosis of microbial keratitis: guidelines for its treatment. J. Evolution Med. Dent. Sci. 2017;6(63):4620-4623, DOI: 10.14260/Jemds/2017/999

\section{BACKGROUND \\ Globally, it is estimated that ocular trauma and corneal ulceration result in 1.5-2 million cases of corneal blindness annually. $90 \%$ of such patients hail from developing countries, where the lesion has now been recognised as a silent epidemic. A National survey by the Government of India (1991-2001) estimated that corneal lesions were responsible for $9 \%$ of blindness in the country.}

Financial or Other, Competing Interest: None.

Submission 13-07-2017, Peer Review 30-07-2017,

Acceptance 01-08-2017, Published 07-08-2017.

Corresponding Author:

V. Sridevi,

Associate Professor,

Department of Ophthalmology,

Rajah Muthiah Medical College \& Hospital,

Annamalai Nagar,

Chidambaram- 608002

E-mail: srithithi@gmail.com

DOI: $10.14260 /$ jemds $/ 2017 / 999$
The epidemiological pattern and causative agents for suppurative corneal ulcer varies significantly from country to country and even from region to region within the same country. It is important to determine the regional aetiology to evolve a comprehensive strategy for diagnosis and treatment of corneal ulcer.

Corneal ulceration is a serious ocular problem that can result in severe visual disability. It is suppurative inflammation of the cornea produced by microorganisms. A careful history and clinical examination is essential to differentiate infectious from non-infectious causes.

Almost any microorganism can invade the corneal stroma if the normal corneal defence mechanisms are compromised. Fungal keratitis is common in elderly men engaged in agricultural work due to increased risk of trauma. Breach of epithelial barrier by even a minor trauma, dust, small metallic foreign bodies, vegetative matter, stone piece, wooden piece, animal matter, soil, cotton fibre predispose the injured eye to develop corneal ulcer. Trauma may breakdown the normal defence mechanism and allow the conjunctival resident flora 
or pathogenic organism from infected lacrimal sac to colonise the damaged tissue to produce corneal ulcer.

Severe corneal ulcer is accompanied by hypopyon. The severity of the ulceration depends upon the virulence of the organism, the integrity of the host defence mechanisms and promptness of appropriate medical attention.

The purpose of this study is to identify factors that influence the outcome of patients with severe infectious corneal ulcers.

A wide spectrum of microbial organisms can produce corneal infections and consequently, the therapeutic strategies adopted for its treatment may be varied. It is important to subject a patient with corneal ulcer to microbiological evaluation in order to attain a definitive diagnosis and to ensure specific therapy for keratitis.

Early recognition, accurate microbiological workup and of appropriate therapy will significantly improve the prognosis. The availability of very potent antibiotics, antifungals and antiamoebic drugs with specific efficacy against different bacterial, fungal and protozoal organisms respectively has resulted in better visual outcome. ${ }^{1}$

The present study is undertaken to evaluate the current concepts of aetiology, microbiological workup and management of suppurative keratitis.

Microbiology plays a major role in providing the various techniques to identify the cause of infection. A constant survey of epidemiological data related to microbiology gives an idea to judge the source of the pathogen and to find out methods to prevent its invasion. ${ }^{2}$ The treatment of microbial keratitis is dependent on the isolation and identification of the aetiological agent and assessment of its susceptibility to the action of various antibiotics in india $3,1,4,5,6,7,8,9$ and abroad. 10

\section{MATERIALS AND METHODS}

This study was undertaken during the period from April 2015 to October 2016. 50 cases of corneal ulcer attending ophthalmic out-patient department of Rajah Muthiah Medical College and Hospital were the subject of this study.

Visual acuity was recorded using Snellen visual acuity chart. Syringing was done to determine the patency of the nasolacrimal duct. The IOP was estimated by using digital pressure above the tarsal plate. Epithelial defect was demonstrated by fluorescein staining the size of the corneal ulcer and the level of the hypopyon is measured with slit lamp.

\section{Inclusion Criteria}

Suspected microbial keratitis

1. Irrespective of age.

2. Irrespective of sex.

\section{Exclusion Criteria}

1. Mooren's ulcer.

2. Neurotrophic ulcer.

3. Herpes simplex keratitis.

\section{Corneal Ulcer with Hypopyon}

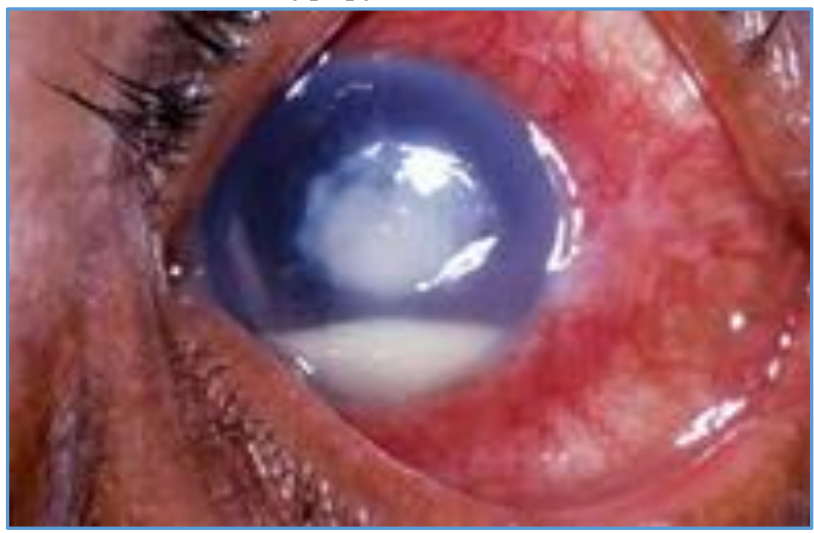

\section{Staphylococcus Aureus}

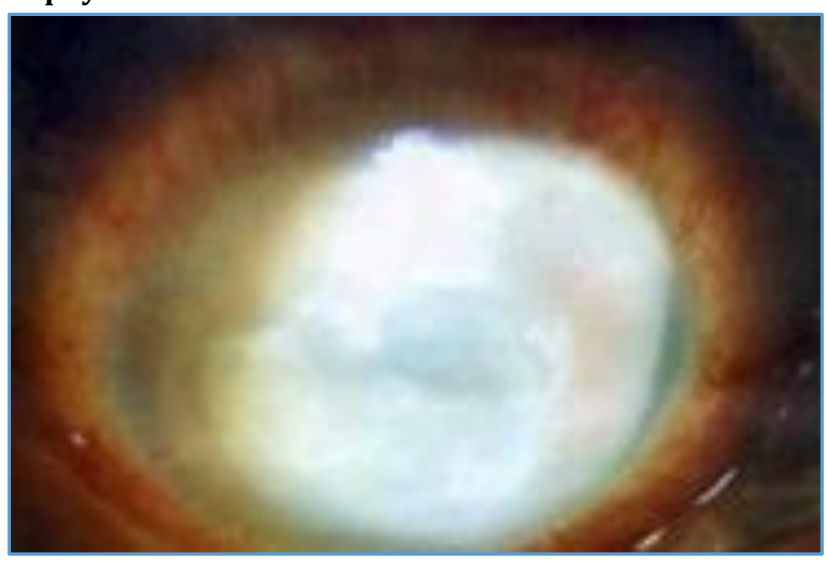

\section{Pseudomonas Aeruginosa}

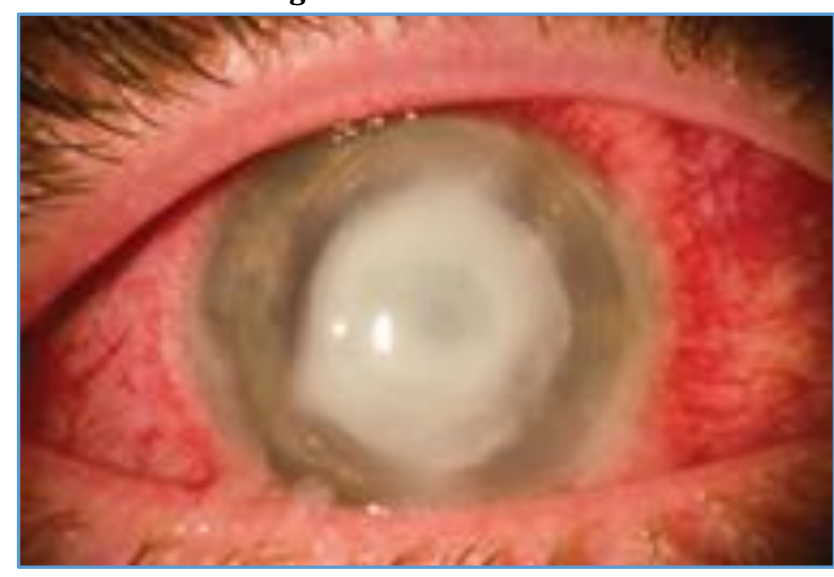

Macular Opacity

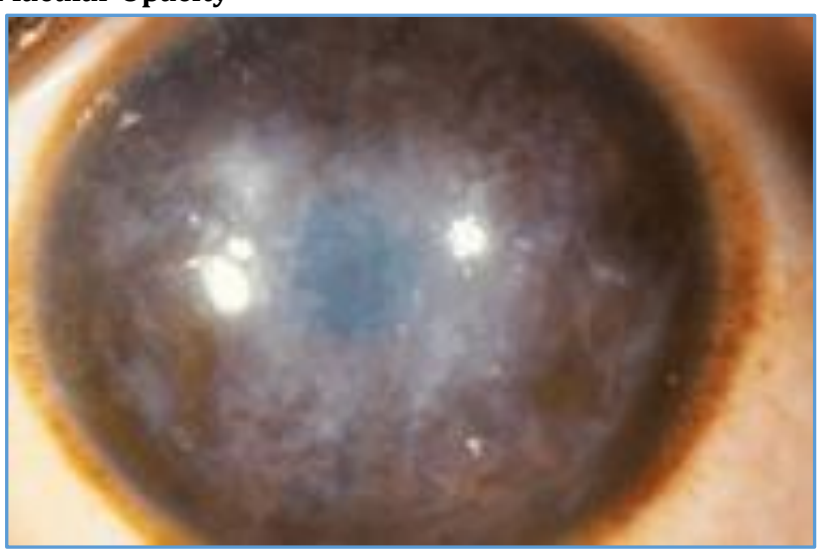




\section{KOH Mount - Showing Branching Septate Fungal Hyphae}

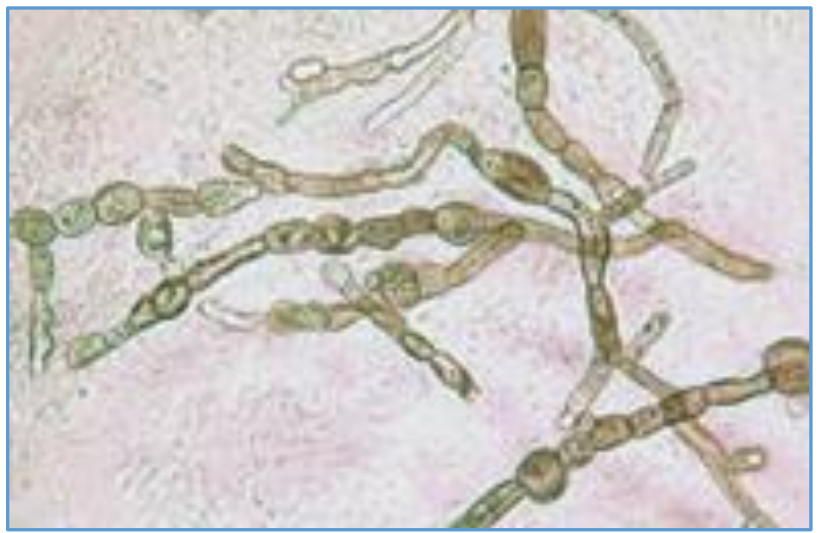

After the instillation of local anaesthetic $5 \mathrm{~g} / \mathrm{L}$ Proparacaine hydrochloride, corneal scraping was obtained aseptically with a sterile No.15 Parker surgical blade from the base and edges of each ulcer.3,1,6,7,11 By using 26-gauge needle, the smear was taken from the margin of ulcer and the specimen collected was sent for preliminary microscopy and culture studies. A portion of each scraping was examined microscopically for the presence of bacteria, fungi or Acanthamoeba by using Gram staining, 10\% KOH and Giemsa staining methods. Another portion was inoculated on to blood agar, chocolate agar, Sabouraud's agar, brain heart infusion broth, in C-shaped streaks and cultured for the potential growth of bacteria, fungi or Acanthamoeba.

For bacterial cultures, the materials were inoculated aerobically at $37^{\circ} \mathrm{C}$ onto blood agar, chocolate agar, and potato dextrose agar (PDA). Cultures on blood agar and chocolate agar was evaluated after 24 hours and 48 hours, and then discarded if there was no growth. The inoculated SDA media were incubated at $27^{\circ} \mathrm{C}$ and were examined daily until three weeks for growth.3,6,7,8 Fungi were identified by their colony characteristics on SDA and by the morphological appearance of the hyphae and spores in Lactophenol cotton blue stain.3,6,7 The growth of the same organism was demonstrated on two or more medias.

The criteria adopted for microbial evaluation was that the sample would be considered positive if any one of the following criteria were met.1,7,8,11

a. The growth of the same organism was demonstrated on two or more medias.

b. The same organism was grown from repeated scrapings.

c. It was consistent with clinical signs.

d. Smear results confirmed the finding from cultures. $3,7,11$

The cultures were examined daily for growth, colony characteristics, pigmentation and rapidity of growth. All results were recorded in master chart. Further the bacteria were tested for their resistance against the following ocular antibiotics: Cefuroxime, Moxifloxacin, Gentamycin Tobramycin, Ceftazidime, Gatifloxacin.

\section{Classification of Causative Organism} Bacterial

\begin{tabular}{|c|c|}
\hline Class & Common Organism \\
\hline \multirow{4}{*}{ Gram-Positive Organism } & Staphylococcus aureus \\
\cline { 2 - 2 } & Staphylococcus albus \\
\cline { 2 - 2 } & Streptococcus pneumonia \\
\cline { 2 - 2 } & Streptococcus viridians \\
\hline
\end{tabular}

\begin{tabular}{|c|c|}
\hline \multirow{4}{*}{ Gram-Positive Bacilli } & Corynebacterium species \\
\cline { 2 - 2 } & Propionibacterium species \\
\cline { 2 - 2 } & Mycobacterium species \\
\cline { 2 - 2 } Gram-Negative Cocci & Nocardia \\
\hline \multirow{4}{*}{ Gram-Negative Bacilli } & Neisseria species \\
\cline { 2 - 2 } & Pseudomonas species \\
\cline { 2 - 2 } & Serratia species \\
\cline { 2 - 2 } & Proteus species \\
\cline { 2 - 2 } & Haemophilus species \\
\hline
\end{tabular}

Fungal

\begin{tabular}{|c|c|}
\hline \multicolumn{1}{|c|}{ Type } & Species \\
\hline \multirow{2}{*}{ Yeast } & Candida \\
\cline { 2 - 2 } & Trichosporon \\
\cline { 2 - 2 } Filamentous, Septated, Non-pigmented & Cryptococcus \\
\cline { 2 - 2 } & Fusarium \\
\cline { 2 - 2 } & Aspergillus \\
\hline \multirow{2}{*}{ Filamentous, Septated, Pigmented } & Penicillium \\
\cline { 2 - 2 } & Curvularia \\
\hline \multirow{2}{*}{ Filamentous, Non-septated } & Alternaria \\
\cline { 2 - 2 } & Mucor \\
\hline
\end{tabular}

Treatment was initiated by debridement of necrotic tissue for better antibiotic penetration. The ulcer was thoroughly scraped and washed with normal saline. Cycloplegics, antibiotics or fortified antibiotics, antifungals, analgesics were given. Patients were advised to report every day to the hospital for review until a clinical healing of ulcer were achieved. Improvement of visual acuity, decrease in ulcer size along with, infiltration, hypopyon, congestion and subjective symptomatic improvement were taken as a positive response to therapy and were recorded.

\section{RESULTS}

In the study of 50 cases of corneal ulcer the following results were obtained. The percentage of fungal keratitis is $54 \%$ proved clinically and by culture and sensitivity while that of bacterial keratitis is $46 \%$ the majority of patients were found in the Age group of less than 50 years male patients showed higher prevalence than female patients most of the patients are agriculturists (64\%). Right eye was affected more (66\%) due to right handedness of the individual. $64 \%$ of the patients presented with history of injury with stick (24\%), paddy husk $(12 \%)$ and thorn $(12 \%) .24 \%$ of patients used topical medication like antibiotics and steroids prior to reporting. $86 \%$ of patients attended our OPD within 5 days of injury. The rest reported after 10 days. The size of the ulcer measured up to $4 \mathrm{~mm}$ in $94 \%$ of patients. The nasolacrimal duct was patent in $90 \%$ of patients. The most common bacteria identify was staphylococcus $16 \%$ and pneumococci $33 \%$. As far as other eye is concerned, $54 \%$ were normal, $20 \%$ showed lens change and $10 \%$ were pseudophakics. The fungi identified were Aspergillus 27\% and Fusarium 73\%.

\section{DISCUSSION}

A variety of factors determine the clinical outcome in microbial keratitis. In our study, corneal ulcer was common in young active adult males (64\%) than females and the same 
incidence was also observed in studies done in Kashmir ${ }^{5}$ South India ${ }^{7}$ and Ghana. ${ }^{11,9}$

The majority of patients were agriculturists which was also observed in studies done in South India ${ }^{7,8}$ Madurai and India. ${ }^{3}$

$24 \%$ of the patients who presented to us with corneal lesion had taken some native treatment or over-the-counter medications from a medical shop in a rural setup which was also observed in a study done in South India.7,8

Streptococcus pneumonia was found to be the most common infecting agent in Nepal and South India ${ }^{1,7,8}$ and Pseudomonas was common in Ghana. ${ }^{9}$

Due to climate changes, Fusarium was known to predominate in Florida, Nigeria, Hong Kong and Singapore. ${ }^{7}$

The percentage of Mycotic keratitis in this series is $62 \%$ which is proved clinically. The percentage of Bacterial keratitis is $38 \%$. Male patients $(64 \%)$ showed higher prevalence than the female patients (36\%) due to higher risk of exposure to injury during field work. Most of the patients are agriculturists (64\%). Right eye was more commonly affected than the left eye, probably due to right handed nature of the individual. In the present study, the patients gave a history of injury either with stick, thorn, paddy husk and other vegetable matter. $24 \%$ of the patients had used topical medications. Those were antibiotics and steroids prior to attending ophthalmic outpatient department.

Treatment success was defined as complete healing of the ulcer within 2 weeks after starting therapy. Most of the patients had come in advanced stages of the disease due to negligence, poor economic condition, ignorance, blind faith in native treatment.

It was observed that most of our patients presented in advanced stage of the disease. The delayed attendance reflects their negligence, poor economic conditions, ignorance of the seriousness and blind faith in native treatment.

The need for proper education of the people especially the rural masses were emphasised in this study. They should be made to realise the importance of early and proper treatment.

Preventive measures were taught to people as a part of our study.

It was emphasised that a proper health care delivery system should be evolved. The necessary medical aid and facilities should be made available to the poor and downtrodden people living in the villages.

People living in cities also should be taught to utilise the existing facilities available to them.

Patients suffering from chronic ocular conditions such as dry eye syndrome, severe blepharitis and chronic dacryocystitis should be monitored closely and forewarned about the development of corneal ulcerations and their complications.

\section{CONCLUSION}

Corneal diseases leave permanent opacities which bring down the visual acuity and may even lead to complete loss of vision.

By looking at these aspects if people are made aware of value of prompt and adequate treatment, there will be significant reduction in the number of corneal blindness cases in our country.

\section{REFERENCES}

[1] Bharathi MJ, Ramakrishnan R, Vasu S, et al. Aetiological diagnosis of microbial keratitis in south India-a study of 1618 cases. Indian J Med Microbiol 2002;20(1):19-24.

[2] Bourcier T, Thomas F, Borderie V, et al. Bacterial keratitis: predisposing factors, clinical and microbial review of 300 cases. Br J Ophthalmol 2003;87(7):8348.

[3] Basak SK, Basak S, Mohanta A, et al. Epidemiological and microbiological diagnosis of suppurative keratitis in gangetic West Bengal, Eastern India. Indian J Ophthalmol 2005;53(1):17-22.

[4] Verenkar MP, Borkar S, Pinto MJW, et al. Study of mycotic keratitis in Goa. Indian Med Microbiol 1998;16(2):58-60.

[5] Bashir G, Shah A, Thokar MA, et al. Bacterial and fungal profile of corneal ulcers: a prospective study. Indian J Pathol Microbiol 2005;48(2):273-7.

[6] Bharathi MJ, Ramakrishnan R, Vasu S, et al. Epidemiological characteristics and laboratory diagnosis of fungal keratitis. A three-year study. Indian J Ophthalmol 2003;51(4):315-21.

[7] Srinivasan M, Gonzales CA, George C, et al. Epidemiology and aetiological diagnosis of corneal ulceration in Madurai, south India. Br J Ophthalmol 1997;81(11):965-71.

[8] Bharathi MJ, Ramakrishnan R, Vasu S, et al. Epidemiology of bacterial keratitis in a referral centre in south India. Indian J Med Microbiol 2003;21(4):239-45.

[9] Leck AK, Thomas PA, Hagan M, et al. Aetiology of suppurative corneal ulcers in Ghana and south India, and epidemiology of fungal keratitis. Br J Ophthalmol 2002;86(11):1211-5.

[10] Wong T, Ormonde S, Gamble G, et al. Severe infective keratitis leading to hospital admission in New Zealand. Br J Ophthalmol 2003;87(9):1103-8.

[11] Hagan M, Wright E, Newman $M$, et al. Causes of suppurative keratitis in Ghana. Br J Ophthalmol 1995;79(11):1024-8. 